\title{
The protective effect of hypothermia on postoperative cognitive deficit may be attenuated by prolonged coronary artery bypass time: Meta-analysis and meta-regression
}

\author{
Valiollah Habibi, ${ }^{1, A, B, D-F}$, Mohammad Reza Habibi ${ }^{2, A, B, D-F}$, Ali Habibi ${ }^{3, B, E, F}$, Amir Emami Zeydi ${ }^{4, A,-F}$ \\ ${ }^{1}$ Department of Cardiac Surgery, Faculty of Medicine, Mazandaran University of Medical Sciences, Sari, Iran \\ ${ }^{2}$ Department of Anesthesiology, Faculty of Medicine, Mazandaran University of Medical Sciences, Sari, Iran \\ ${ }^{3}$ Student Research Committee, Faculty of Medicine, Mazandaran University of Medical Sciences, Sari, Iran \\ ${ }^{4}$ Department of Medical-Surgical Nursing, Nasibeh School of Nursing and Midwifery, Mazandaran University of Medical Sciences, Sari, Iran \\ A - research concept and design; $\mathrm{B}$ - collection and/or assembly of data; $\mathrm{C}$ - data analysis and interpretation; \\ $D$ - writing the article; $E$ - critical revision of the article; $F$ - final approval of the article
}

Address for correspondence

Mohammad Reza Habibi

E-mail: mohammadreza.habibi@gmail.com

\section{Funding sources}

None declared

\section{Conflict of interest}

None declared

Received on November 21, 2017

Reviewed on December 16, 2018

Accepted on May 1, 2020

Published online on October 16, 2020

\begin{abstract}
There is controversy about whether hypothermia during coronary artery bypass grafting (CABG) surgery is effective in reducing postoperative cognitive deficit (POCD). The objective of this study was to determine the effect of hypothermia on POCD and to undertake a meta-regression to determine whether moderator variables mediate the relationship between hypothermia and POCD. We searched the Web of Science, PubMed database, Scopus, and the Cochrane Library database (up to June 2017), and systematically reviewed a list of retrieved articles. Our final review includes only randomized controlled trials (RCTs) that compared administration of hypothermia $\left(34^{\circ} \mathrm{C}\right)$. Statistical analysis of the risk ratio (RR) and corresponding $95 \%$ confidence interval (95\% Cl) was used to report the overall effect. Mantel-Haenszel risk ratio (MH RR) and corresponding 95\% Cl was used to report the overall effect and meta-regression analysis. Eight RCTs were included in this study, with a total of 1,474 patients. The POCD occurred in $36.06 \%$ of all cases. A wide range of hypothermia $\left(28-34^{\circ} \mathrm{C}\right)$ did not reduce the occurrence of $P O C D\left(R R=0.983\left(95 \%(\mathrm{Cl}=0.881-1.143) ; Z=-0.304 ; \mathrm{P}=0.761 ; \mathrm{I}^{2}=38 \%\right)\right.$. Shorter CPB time reduced the occurrence of POCD (MH log risk ratio $=-0.011(95 \% \mathrm{Cl}=-0.021--0.0008)$; $Z=-2.123 ; P=0.033)$. Postoperative cognitive deficit is a common event among CABG patients. Contrary to deep hypothermia, mild hypothermia was significantly effective in reducing the risk of POCD. The neuroprotective effect of hypothermia on POCD may be attenuated by prolonged cardiopulmonary bypass (CPB) time.
\end{abstract}

Key words: coronary artery bypass grafting, meta-analysis, hypothermia, postoperative cognitive deficit
Cite as

Habibi V, Habibi MR, Habibi A, Emami Zeydi A. The protective effect of hypothermia on postoperative cognitive deficit may be attenuated by prolonged coronary artery bypass time: Meta-analysis and meta-regression. Adv Clin Exp Med. 2020;29(10):1211-1219. doi:10.17219/acem/121920

DOI

10.17219/acem/121920

Copyright

Copyright by Author(s)

This is an article distributed under the terms of the

Creative Commons Attribution 3.0 Unported (CC BY 3.0)

(https://creativecommons.org/licenses/by/3.0/) 


\section{Introduction}

Postoperative cognitive deficit (POCD) has long been understood as a major complication after cardiac surgery. ${ }^{1,2}$ Despite the fact that reported occurrence of POCD varies widely across studies, coronary artery bypass grafting (CABG) with the use of cardiopulmonary bypass (CPB) is associated with an increased risk of morbidity and mortality. ${ }^{3,4}$ The underlying cause of POCD has not yet been properly identified. ${ }^{5}$ It is probable that microemboli are responsible for brain injury after $\mathrm{CPB}$ surgery. ${ }^{6}$

Hypothermia is a widely used protocol to reduce cognitive deficit after CPB surgery. ${ }^{7}$ It reduces cerebral metabolic rate as well as the inflammatory response and apoptosis. ${ }^{8}$ Several studies investigated the effect of hypothermia on cognitive deficit among patients with CABG and they did not find any advantage of hypothermia over normothermia for improving cognitive deficit, ${ }^{4,9-14}$ resulting in ambiguity in terms of the role of temperature management during CPB surgery. Therefore, clinical practice guideline of temperature management advised that arterial outlet blood temperature should be limited to below $37^{\circ} \mathrm{C}$ to avoid cerebral hyperthermia. Although the avoidance of hyperthermia was emphasized, no threshold temperature during hypothermia is recommended due to the insufficient published evidence. ${ }^{15}$ Therefore, a hypothesis may be advanced that there may be other variables that need to be explored.

Due to the effect of hypothermia on neuroprotection, the widespread use of this technique and inconclusive findings from randomized controlled trials (RCTs), our aim was to systematically review and summarize evidence relevant to the effect of hypothermia on the occurrence of POCD and perform a meta-regression to discover whether moderator variables mediate the relationship between hypothermia and POCD.

\section{Methods}

\section{Data sources}

The PubMed, the Web of Science, Scopus, and the Cochrane Library databases were searched for clinical trials up to June 2017, using the search terms: ("coronary artery bypass surgery" OR "cardiopulmonary bypass surgery" OR "thoracic surgery" OR "cardiovascular surgery" OR "cardiac surgery" OR "CABG") AND ("cognitive dysfunction" OR "cognitive function" OR "cognitive decline" OR "neurocognitive") AND ("temperature" OR "*thermia" OR "*thermic" OR "body temperature" OR "perfusion temperature" OR "hypothermic cardiopulmonary bypass surgery" OR "rewarming") AND ("randomized controlled trial" OR "controlled clinical trial" OR "randomized" OR "randomly" OR "trial"). The reference lists of the retrieved studies including systematic reviews and meta-analyses were also checked.

\section{Study selection}

Only RCTs were incorporated into this meta-analysis in which patients undergoing $\mathrm{CPB}$ surgery received hypothermic $\left(<34^{\circ} \mathrm{C}\right)$ and normothermic $\left(>34^{\circ} \mathrm{C}\right) \mathrm{CPB}$ surgery. We excluded non-English-language studies as well as studies that did not administer the neuropsychological test battery and studies that did not measure cognitive deficit after CPB surgery.

\section{Data extraction and clinical endpoint}

The occurrence of any cognitive deficit following CPB surgery (a decline $>1$ standard deviation (SD) in the postoperative score in comparison to the preoperative score) was defined as primary outcome. ${ }^{16}$ The decline must be verified using the 1 or more neuropsychological tests. The occurrence of POCD in time periods within the first 30, 30-90 and 90-360 days were defined as the very early, early and late occurrence of cognitive deficit, respectively. ${ }^{1}$

Studies that met the inclusion criteria were assessed by one reviewer and another reviewer verified the studies. The POCD in intervention and control groups was the main outcome. The potential moderator variables including male gender [\%], age [years], temperature thresholds $\left[{ }^{\circ} \mathrm{C}\right]$, mean duration of $\mathrm{CPB}$ [min], hypertension (HTN) [\%], diabetes mellitus (DM) [\%], reduced left ventricle ejection fraction (rLVEF) [\%], follow-up period [days], and loss to follow-up [\%] were collected. Hypothermia can be categorized as mild and deep hypothermia. A $3^{\circ} \mathrm{C}$ difference between normothermic and hypothermic group is considered as mild hypothermic protocol and more than $3^{\circ} \mathrm{C}$ difference is defined as deep hypothermic protocol. Disagreements between authors were resolved through discussion. The Jadad five-point scale was used to assess the quality of RCT; this scale scores a maximum of 2 points for blinding, 2 points for randomization and 1 point for the description of withdrawals and drop-outs (that is, patients lost to follow-up). ${ }^{16}$ The Q and I ${ }^{2}$ statistics were used to assess the statistical heterogeneity among the included RCTs. Heterogeneity was considered for variables with $\mathrm{Q}$ statistics $(\mathrm{p}<0.05)$. It means that the amount of total variance is more than we would expect based on within-study error, so random effect model was presumed. A random-effect model was used in meta-regression because there was significant between-study variation. ${ }^{17}$ The degree of heterogeneity was determined using $\mathrm{I}^{2}$ statistics and it also represents the proportion of variation in treatment which is independent from sampling error. The $\mathrm{I}^{2}$ statistics result between $30 \%$ and $60 \%$ is moderately heterogeneous. ${ }^{18}$ The relationship between 1 or more moderators and a dependent variable was assessed using meta-regression. ${ }^{19}$ Effect size as dependent variable and moderator variable as independent variable were analyzed to identify potential predictors of effect size. Q-model statistics with $\mathrm{p}<0.05$ showed that the relationship between 
moderator variable and treatment effect is stronger than we would expected by chance. Variables with $\mathrm{Z}$ statistics $(\mathrm{p}<0.05)$ were interpreted as their slope is probably not 0 , and the treatment effect is more effective when moderator variable changes. ${ }^{17}$ Mantel-Haenszel $(\mathrm{MH})$ log risk ratio was regressed on the following variables: between-group proportion difference of male gender [\%], between-group mean difference of age [years], CPB time [min], and temperature threshold [ ${ }^{\circ} \mathrm{C}$ ]. Hypertension [\%], DM [\%] and rLVEF [\%] were not reported in all trials. Difference was defined as the difference between normothermia and hypothermia groups (normothermia - hypothermia). Negative value of $\mathrm{MH}$ log risk ratio is in favor of the neuroprotective effect of hypothermia. Data are presented as a mean (SD) for continuous variables and as proportions (\%) for categorical variables. Statistical analyses were conducted using the Comprehensive Meta-Analysis v. 2 statistical software package (Biostat Inc, Englewood, USA). Dichotomous results were analyzed using the Mantel-Haenszel method. The risk ratio (RR) and its 95\% confidence interval (95\% CI) were calculated.

\section{Results}

\section{Search results}

From a total of 715 citations identified, 29 articles were fully evaluated. A total of 1,747 patients in 8 RCTs met the inclusion criteria and all of them were included in the final analyses. ${ }^{20-27}$ Steps of the search strategy are presented in Fig. 1.

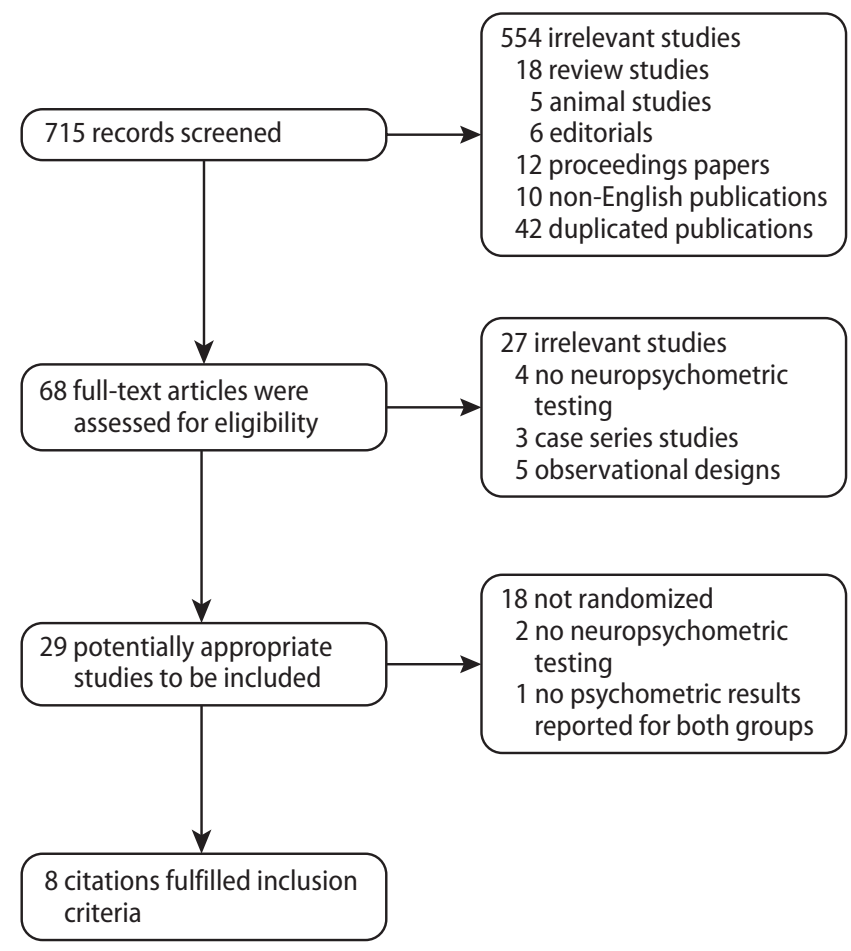

Fig. 1. Flow diagram of the selection process of eligible articles

\section{Study characteristics}

All RCTs scored above moderate and 2 studies scored high on the Jadad scale (Table 1). The Wechsler Adult Intelligence Scale was the most frequently used scale to assess the severity of POCD. The overall occurrence of POCD was $36.06 \%$. The occurrence of POCD was $48.23 \%, 15.21 \%$ and $48.49 \%$ for very early, early and late periods, respectively. Male patients had a higher proportion than female patients in all RCTs. Mean surgery time (CPB) ranged from $71 \mathrm{~min}$ to $127.5 \mathrm{~min}$ and surgery time difference between hypothermic and normothermic groups was up to $27.3 \mathrm{~min}$. No significant between-group difference was reported in the baseline characteristics of patients (except for CPB time in the study by Plourde et al.). The key characteristics of the included studies are presented in Table 1. Data and conclusions are summarized in Table 2.

\section{Overall effect of hypothermia}

The overall effect of hypothermia was estimated through a meta-analysis. The effect of hypothermia on POCD was calculated through MH RR as 0.983 (95\% CI = 0.8811.098). The MH RRs for very early, early and late cognitive deficit were 1.004 (95\% CI $=0.883-1.143), 0.968$ (95\% CI $=0.722-1.299)$ and 0.887 (95\% CI $=0.656-1.198)$ (Fig. 2). The findings of the pooled analyses are summarized in Fig. 2. The overall heterogeneity of the RCTs was estimated as 38\% (Q-model $=20.912 ; \mathrm{p}=0.075)$. A funnel plot for publication bias analysis is not presented due to the fact that it is not recommended with analysis of fewer than 10 studies. ${ }^{19}$

\section{Meta-regression}

The overall effect of moderator variables was estimated through meta-regression analyses. The model was significant for the association of CPB time difference as well as temperature difference with cognitive deficit. Betweengroup temperature difference was calculated through $\mathrm{MH} \log \mathrm{RR}$ as 0.053 (95\% CI = 0.007-0.098); $\mathrm{Z}=2.302$, $\mathrm{p}=0.021, \mathrm{Q}$-model $=5.303$, degree of freedom $(\mathrm{df})=1$, $\mathrm{p}=0.021$ (Fig. 3A). The mild temperature difference (about $3^{\circ} \mathrm{C}$ ) is significantly associated with a lower rate of POCD in comparison with deep hypothermia $\left(>3^{\circ} \mathrm{C}\right)$ (Fig. $\left.3 \mathrm{~A}\right)$. Figure $3 \mathrm{~B}$ shows that the $\mathrm{MH} \log \mathrm{RR}$ for $\mathrm{CPB}$ time difference between groups was -0.011 (95\% CI $=-0.021-$ $-0.0008) ; \mathrm{Z}=-2.123, \mathrm{p}=0.033, \mathrm{Q}$-model $=4.509, \mathrm{df}=1$, $\mathrm{p}=0.033$ (Fig. 3B) and it shows that shorter CPB time is significantly associated with a lower rate of POCD in either group.

Stepwise regression analyses were performed to examine the association between $\mathrm{MH}$ log risk ratio and potential predictors including temperature and CPB time difference. The $\mathrm{R}^{2}$ was $43.8 \%$. Regression model was significant, $\mathrm{R}=0.693, \mathrm{R}^{2}=0.431, \mathrm{p}=0.006$. The $\mathrm{CPB}$ time was 
Study name Subgroup within study

McLean et al. 1994 Mora et al. 1996 Regragui et al. 1996 Heyer et al. 1997 Boodhwani et al. 2007

Kaukinen et al. 2000 Nathan et al. 2007

McLean et al. 1994 Mora et al. 1996 Heyer et al. 1997 Plourde et al. 1997 Kaukinen et al. 2000 Boodhwani et al. 2007 Nathan et al. 2007

early
early
early
early
early
early
late
late
late
very early
very early
very early
very early
very early
very early
very early
very early
overall

Statistics for each study

$\begin{array}{cccrr}\begin{array}{c}\text { MH risk } \\ \text { ratio }\end{array} & \begin{array}{c}\text { lower } \\ \text { limit }\end{array} & \begin{array}{c}\text { upper } \\ \text { limit }\end{array} & \text { Z-value } & \text { p-value } \\ 1.974 & 0.708 & 5.505 & 1.300 & 0.194 \\ 0.729 & 0.169 & 3.135 & -0.425 & 0.671 \\ 1.076 & 0.781 & 1.482 & 0.447 & 0.655 \\ 0.889 & 0.507 & 1.560 & -0.411 & 0.681 \\ 0.458 & 0.164 & 1.282 & -1.487 & 0.137 \\ 0.968 & 0.722 & 1.299 & -0.215 & 0.830 \\ 1.222 & 0.874 & 1.708 & 1.172 & 0.241 \\ 0.745 & 0.484 & 1.145 & -1.342 & 0.179 \\ 0.887 & 0.656 & 1.198 & -0.783 & 0.433 \\ 0.886 & 0.638 & 1.230 & -0.724 & 0.469 \\ 1.064 & 0.653 & 1.734 & 0.249 & 0.804 \\ 1.122 & 0.899 & 1.400 & 1.019 & 0.308 \\ 1.000 & 0.684 & 1.462 & 0.000 & 1.000 \\ 2.143 & 1.212 & 3.788 & 2.621 & 0.009 \\ 1.091 & 0.845 & 1.410 & 0.670 & 0.503 \\ 0.694 & 0.482 & 0.998 & -1.970 & 0.049 \\ 1.004 & 0.883 & 1.143 & 0.067 & 0.946 \\ 0.983 & 0.881 & 1.098 & -0.304 & 0.761\end{array}$

\section{$\mathrm{MH}$ risk ratio and $95 \% \mathrm{Cl}$}

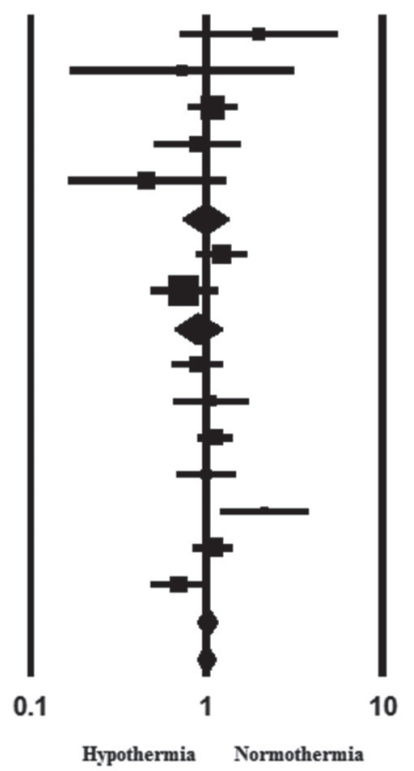

$\mathbf{I}^{2}$

$\begin{array}{cccccc}\text { Z-value } & \text { P-value } & \text { Q-value } & \mathbf{d f}(\mathbf{Q}) & \text { P-value } & \mathbf{I}^{\mathbf{2}} \\ 0.067 & 0.946 & 12.749 & 6 & 0.047 & 52.938 \\ -0.215 & 0.830 & 4.534 & 4 & 0.339 & 11.772 \\ -0.783 & 0.433 & 4.148 & 1 & 0.042 & 75.89 \\ -0.304 & 0.761 & 20.912 & 13 & 0.075 & 37.835\end{array}$

Fig. 2. Comparison of patients on hypothermia and normothermia
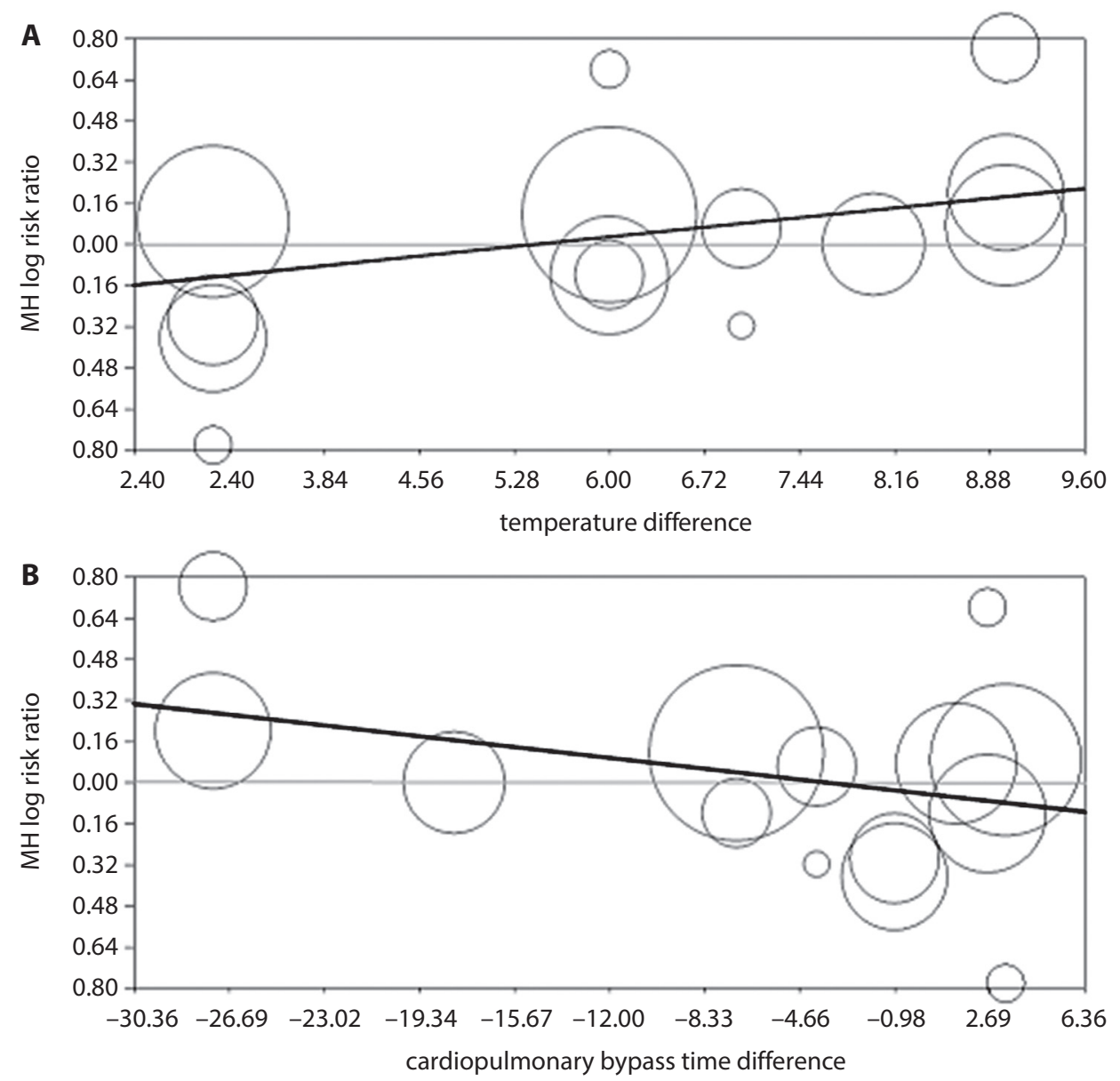

Fig. 3. A. Plot of MH log risk ratio against temperature difference between groups. Positive values in the horizontal axis indicate a greater mean or proportion for the normothermic group (difference $=$ normothermia

- hypothermia). Negative values in the vertical axis favor the neuroprotective effect of hypothermia (below the horizontal grey line). B. Plot of MH log risk ratio against cardiopulmonary bypass time difference between groups. Negative values in the horizontal axis indicate a greater mean or proportion for the hypothermic group (difference $=$ normothermia - hypothermia). Negative values in the vertical axis favor the neuroprotective effect of hypothermia (below the horizontal grey line) 


\begin{tabular}{|c|c|c|c|c|c|c|c|c|c|c|c|c|}
\hline 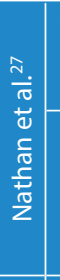 & 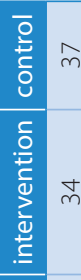 & $\frac{n}{8}$ & $\infty \sqrt{\pi}$ & in $\underset{\alpha}{\widehat{\alpha}}$ & $\stackrel{\infty}{-} \stackrel{\oslash}{d}$ & 응 & $\infty$ & 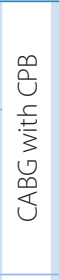 & 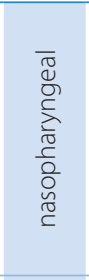 & 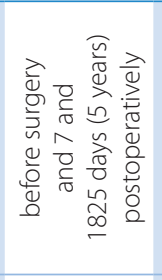 & 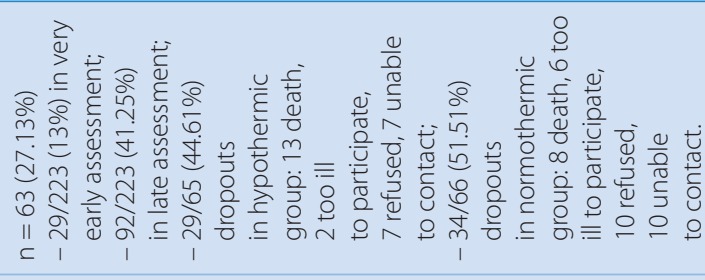 & in \\
\hline 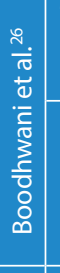 & 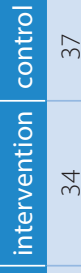 & $\stackrel{\substack{m \\
m}}{\stackrel{m}{m}}$ & 赵 & $\stackrel{\cong}{=} \underset{\infty}{\mathscr{\infty}}$ & F命 & 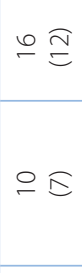 & 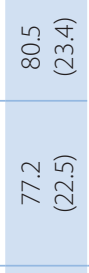 & 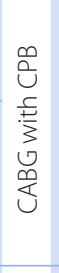 & 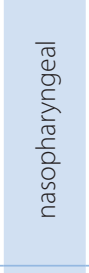 & 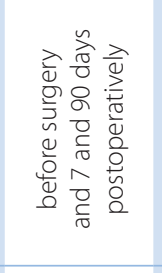 & 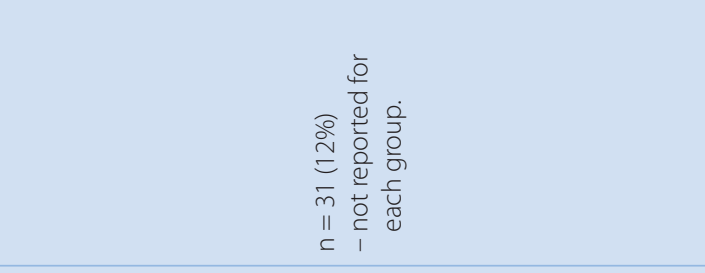 & $m$ \\
\hline 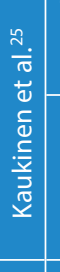 & 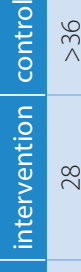 & 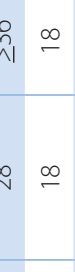 & 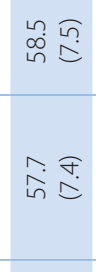 & 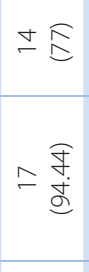 & 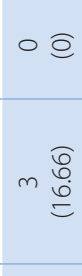 & 1 & 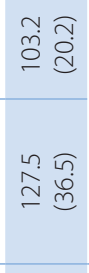 & 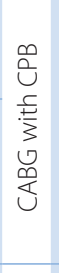 & 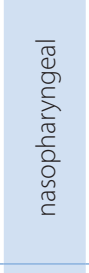 & 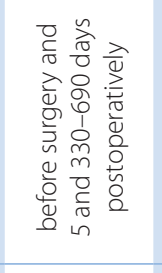 & 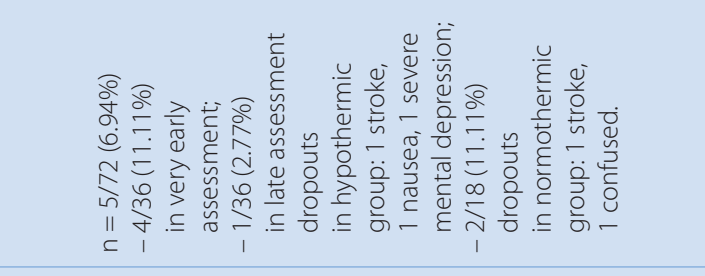 & $m$ \\
\hline 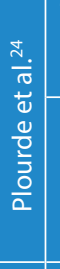 & 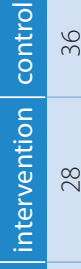 & 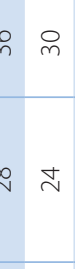 & 官 & 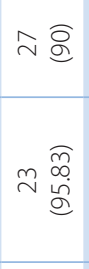 & 1 & 1 & $\overline{\bar{d}}$ & 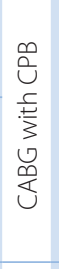 & 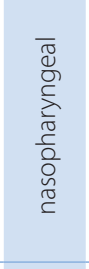 & 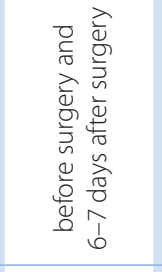 & 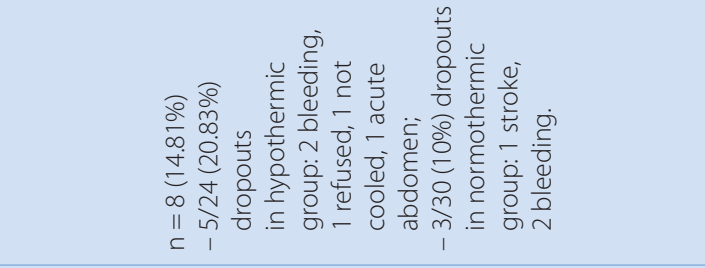 & in \\
\hline 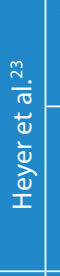 & 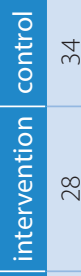 & 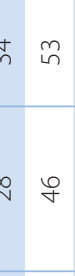 & in & of & 1 & 1 & 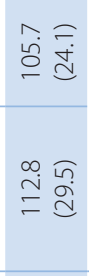 & 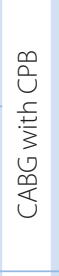 & 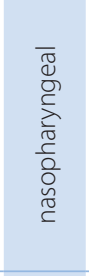 & 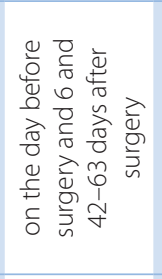 & 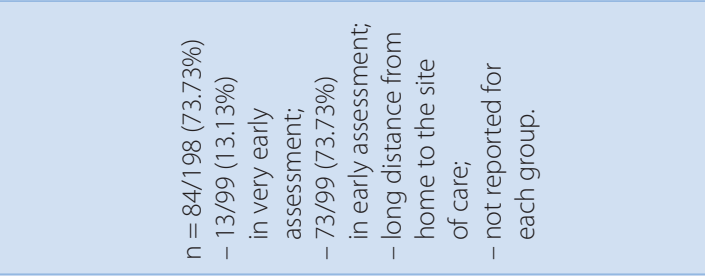 & $m$ \\
\hline 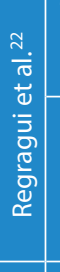 & 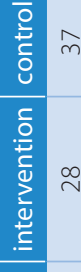 & $\frac{i}{n}$ & 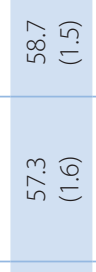 & ৯ & $-\underset{d}{\mathbb{f}}$ & 1 & 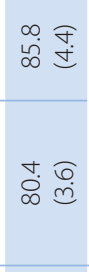 & 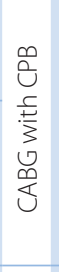 & 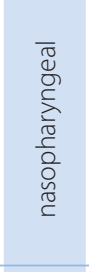 & 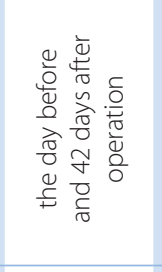 & 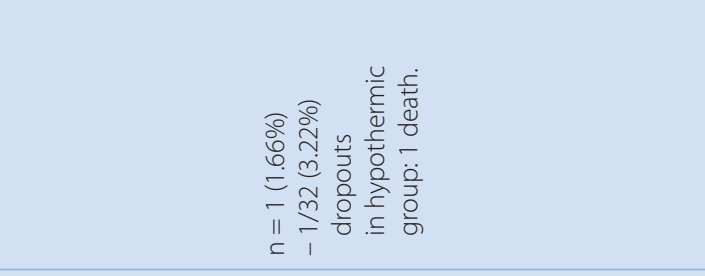 & $m$ \\
\hline 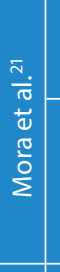 & 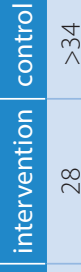 & in & $\overline{0} \bar{E}$ & $\bar{n} \stackrel{\nwarrow}{\triangleq}$ & $\stackrel{ }{\stackrel{d}{d}}$ & 1 & $\infty \approx \widetilde{\widetilde{d}}$ & 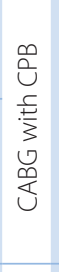 & $\begin{array}{l}\frac{\overline{0}}{0} \\
\frac{\pi}{0}\end{array}$ & 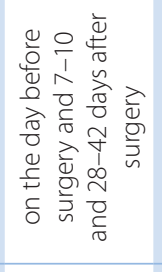 & 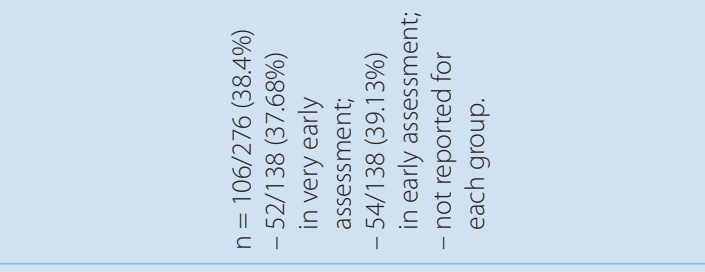 & $\nabla$ \\
\hline 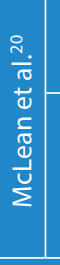 & 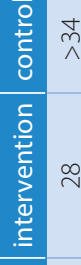 & $\stackrel{N}{N} \stackrel{\infty}{\wedge}$ & is & I & 1 & m衰 & 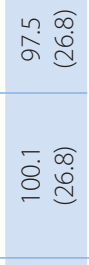 & 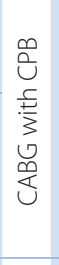 & 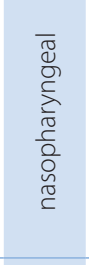 & 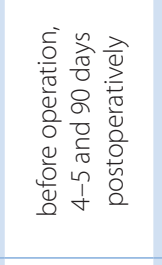 & 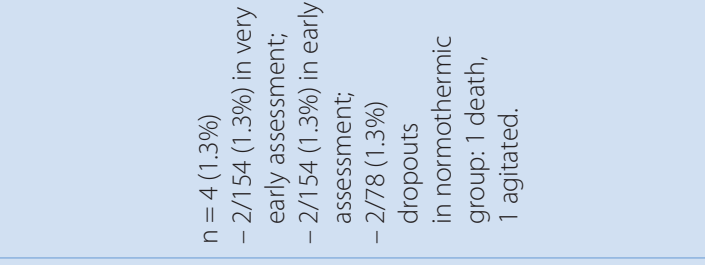 & $m$ \\
\hline 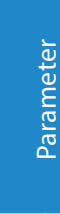 & 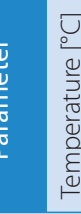 & 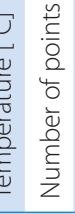 & 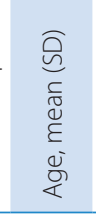 & $\frac{\frac{\partial}{\tilde{v}}}{\frac{\tilde{v}}{\tilde{N}}}$ & $\begin{array}{l}\frac{\tilde{u}}{ \pm} \\
\frac{0}{.0} \\
\overline{0}\end{array}$ & 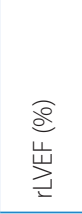 & 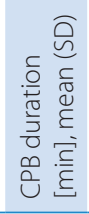 & 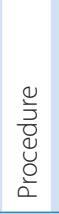 & 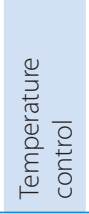 & 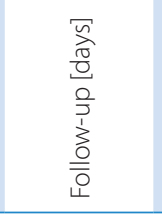 & 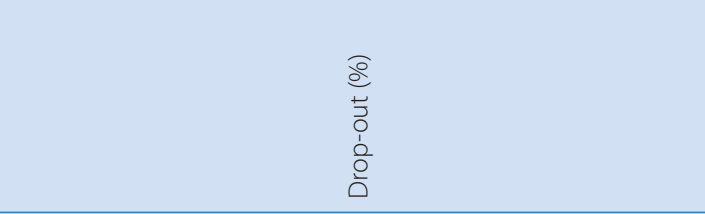 & $\begin{array}{l}0 \\
\overline{0} \\
\underline{n} \\
0 \\
\frac{\pi}{0} \\
\frac{0}{9}\end{array}$ \\
\hline
\end{tabular}




\begin{tabular}{|c|c|c|c|c|c|c|c|c|}
\hline & 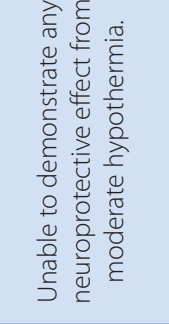 & 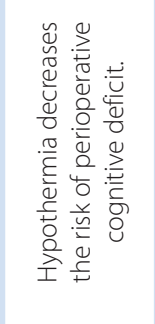 & 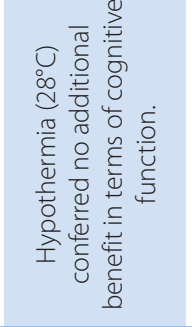 & 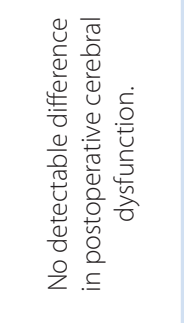 & 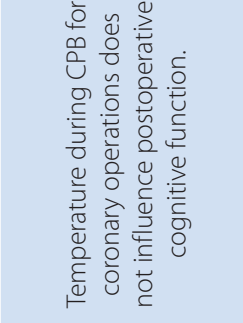 & 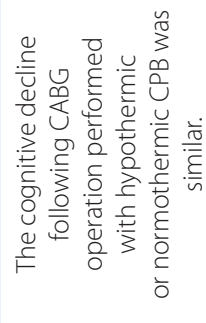 & 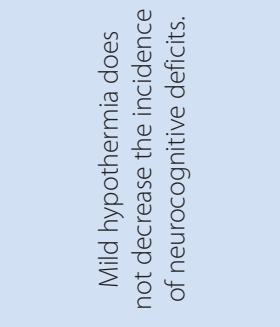 & 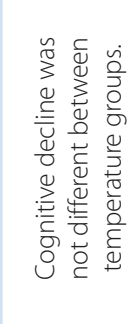 \\
\hline & 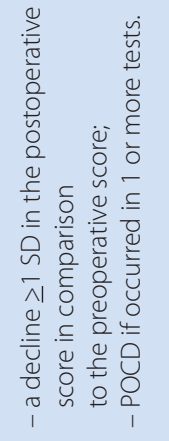 & 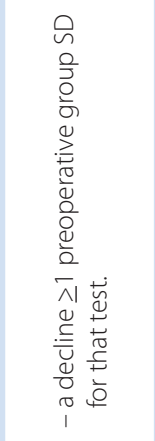 & 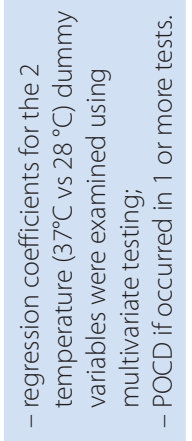 & 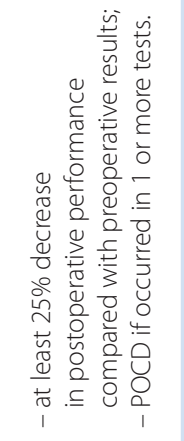 & 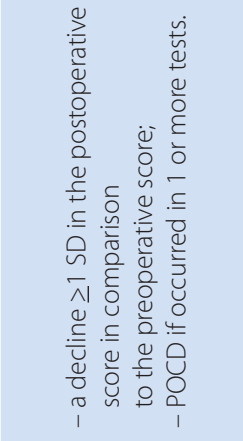 & 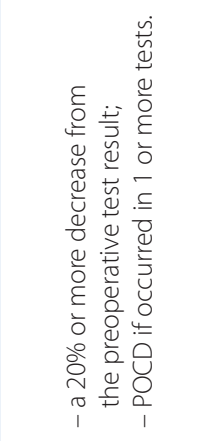 & 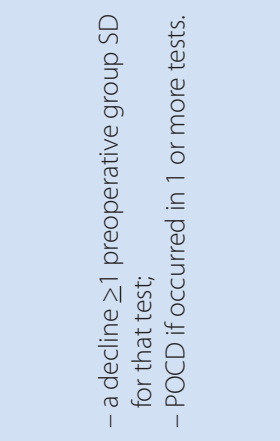 & 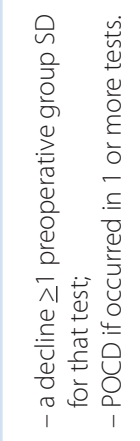 \\
\hline & 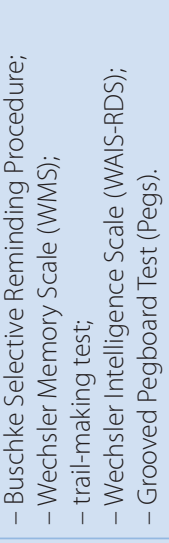 & 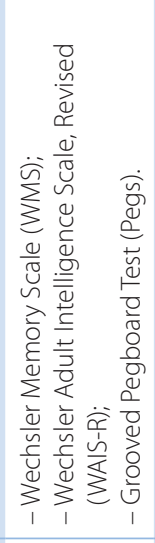 & 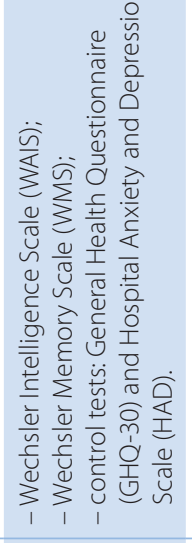 & 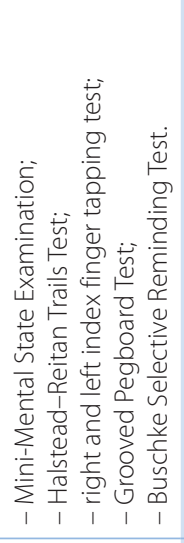 & 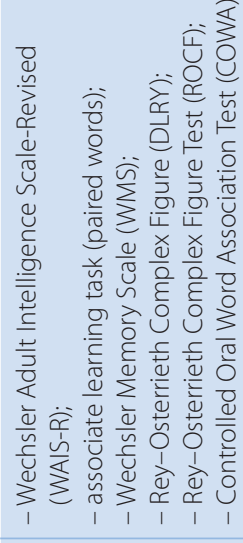 & 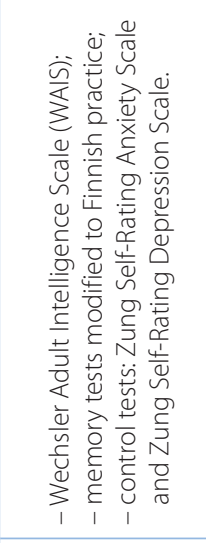 & 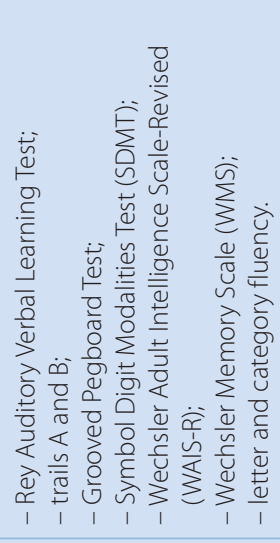 & 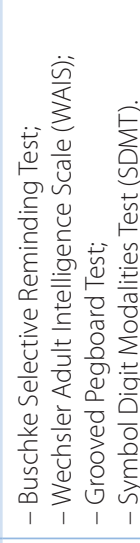 \\
\hline & 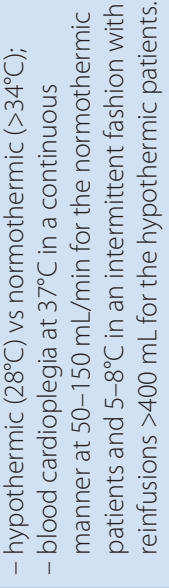 & 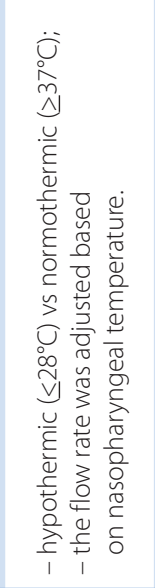 & 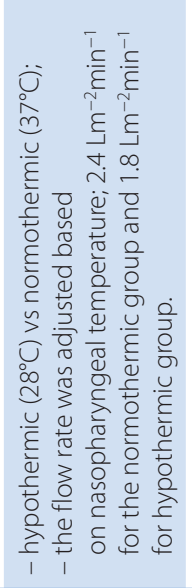 & 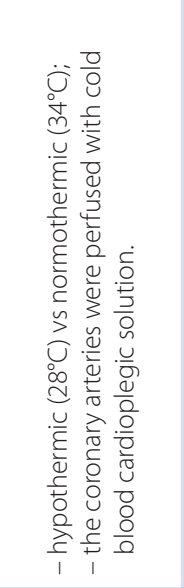 & 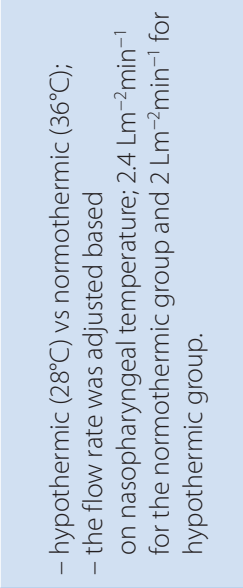 & 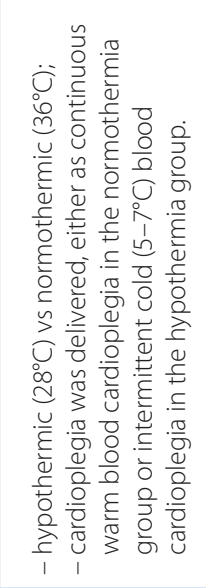 & 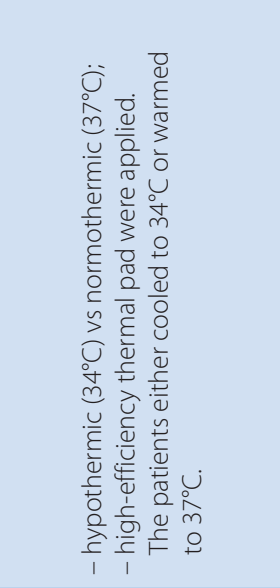 & 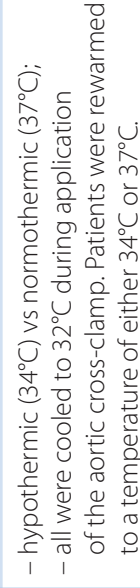 \\
\hline & 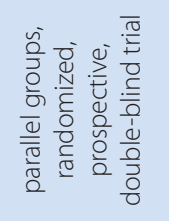 & 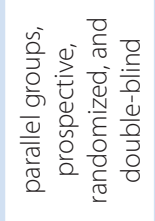 & 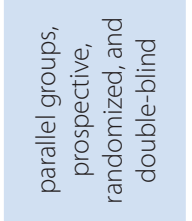 & 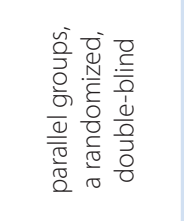 & 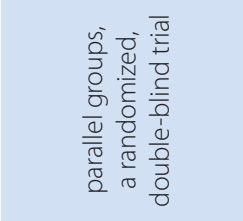 & 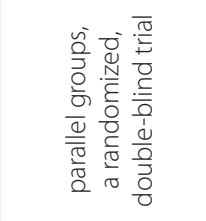 & 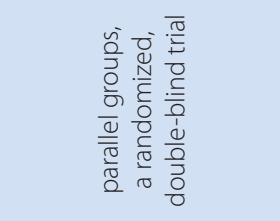 & 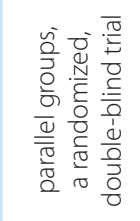 \\
\hline & 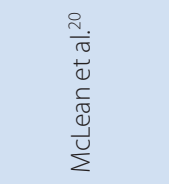 & 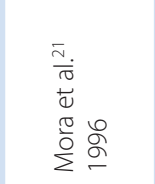 & $\frac{\pi}{\pi} \frac{\pi}{\pi}$ & 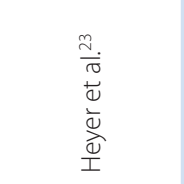 & $\begin{array}{l}\frac{0}{\pi} \\
\frac{\pi}{0} \\
\frac{0}{0} \\
\frac{0}{0} \\
\frac{0}{0}\end{array}$ & & 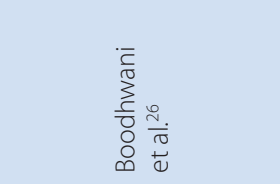 & 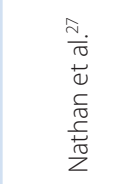 \\
\hline
\end{tabular}


significantly associated with $\mathrm{MH} \log$ risk ratio - unstandardized B CPB time was $-0.62(0.018 ; \mathrm{p}=0.006)$ and unstandardized B constant was -0.157 .

\section{Sensitivity analysis}

Only Plourde et al. reported a significant difference of $\mathrm{CPB}$ time between groups (CPB time of hypothermic group was longer than that of normothermic group). ${ }^{24}$ Slope was significant after exclusion of the study by Plourde et al. and it resulted in a reduction from -0.011 to -0.045 , meaning that the association between $\mathrm{CPB}$ time and POCD was not influenced by the results of the study conducted by Plourde et $\mathrm{al}^{24}$. There are no influential studies in any of the analyses estimated from "Remove-One" sensitivity analysis.

\section{Discussion}

To date, this is the first meta-regression to evaluate the association between the neuroprotective effect of hypothermia and moderator variables. We demonstrated that shorter CPB time had a favorable influence on POCD. This finding may have critical implications for the therapeutic management of patients undergoing CABG surgery. ${ }^{1}$

It is well-recognized that hypothermia is neuroprotective. ${ }^{28}$ Hypothermia provides cerebral protection against ischemia, reduces brain metabolism under hemodynamic instability, and inhibits free radical and oxidative enzymatic activities and attenuation of the excitatory and inhibitory neurotransmitters, resulting in a protective balance against brain damage. ${ }^{13}$ In detail, extracellular amino acids play a pivotal role in neural damage. ${ }^{28,29}$ However, glutamate, aspartate, gamma-aminobutyric acid, taurine, glycine, and alanine showed a significant increase in response to the ischemia in normothermia. Therefore, inhibition of excessive effluxes of both excitatory and inhibitory amino acids contributes to the neuroprotective effect of hypothermia against ischemia.

In harmony with other reviews on the association of hypothermia and POCD, , $4,12-14$ our meta-analysis showed that a wide range of hypothermia $\left(28-34^{\circ} \mathrm{C}\right)$ does not reduce POCD after CABG surgery. However, there is no statistically significant difference between the 2 groups, risk ratio for very early (1.004), early (0.968) and late (0.887), showing that the trend is compatible with that reported by Brown et al. ${ }^{6}$ They demonstrated that there is a rapid decline in the embolic load as time from surgery increases (particularly in the first few days after surgery). In our study, there is a trend toward a lower occurrence of cognitive deficit among patients after $\mathrm{CPB}$ surgery in hypothermic group (35.45\% compared to $36.66 \%$ ), suggesting that a moderator variable may interfere with a significant decrease in the POCD in hypothermic group.

Meta-regression demonstrated shorter CPB time is associated with a lower occurrence of POCD in either group, meaning that $\mathrm{CPB}$ surgery time is an independent predictor of POCD. In the regression model, patients with longer surgery time had a significantly increased risk for the occurrence of POCD. Bearing in mind that only 2 (25\%) studies had a shorter surgery time for the hypothermic group, it may explain why the findings of these studies have been inconclusive across all previous reviews. Figure 3B shows that the neuroprotective effect of hypothermia can only resist up to 4 min longer mean duration of normothermic $\mathrm{CPB}$. This finding is supported by other studies, indicating that an increase in $\mathrm{CPB}$ surgery time is associated with an embolic load increase. ${ }^{6,30,31}$ The key role of CPB surgery time in association with the risk of microemboli development and POCD has been well-discussed in the cardiac surgery studies. ${ }^{30-32}$ Brown et al. reported that a $30.5 \%$ increase in the embolic load is contributed by each 60 -minute increase in CPB surgery time. ${ }^{6}$ Patients with valve plasty surgery experience even more severe embolic load $(145.3 \% / h)$. In this line, Salis et al. showed that a 30-minute increment in CPB surgery time is a significant independent risk factor for postoperative death $(\mathrm{OR}=1.57) .{ }^{33}$ In addition, Bucerius et al. reported that $\mathrm{CPB}$ surgery time longer than $120 \mathrm{~min}$ is an independent risk factor for stroke $(\mathrm{OR}=1.42) .{ }^{34}$ Therefore, it has to be said that postoperative outcome of patients with CABG surgery is considerably associated with CPB surgery time.

In this review, absolute mean $\mathrm{CPB}$ surgery time difference between normothermic and hypothermic groups was up to 27.3 min and mean $\mathrm{CPB}$ surgery time ranged from $71 \mathrm{~min}$ to $127.5 \mathrm{~min}$. However, all RCTs (except that of Plourde et al. ${ }^{24}$ ) reported that the average of CPB surgery time does not differ significantly between groups. Hypothermic groups experienced greater mean CPB surgery time in 6 studies. ${ }^{20-25,27}$ Therefore, the effect of hypothermia on POCD may be confounded by the great impact of prolonged $\mathrm{CPB}$ time on emboli formation. Also, mean statistics can be highly affected by the extreme values and it is not an appropriate statistic for highly skewed distributions. An analysis of 5,000 patients undergoing CABG surgery showed that the distribution of $\mathrm{CPB}$ duration is positively skewed $(0.18)$ and data is dispersed (coefficient of variation $(\mathrm{CV})=40 \%$ ), showing that $25 \%$ of surgeries lasted longer than $135 \mathrm{~min}$ (up to $643 \mathrm{~min}$ ). ${ }^{33}$ In this review, each 30-minute increment in $\mathrm{CPB}$ surgery time increases the risk of death 1.53 times, which shows that $\mathrm{CPB}$ surgery time is closely related to the final outcomes such as death, stroke and POCD. In the included studies, there is a high dispersion in data due to the fact that $\mathrm{CV}$ of CPB surgery time ranged from $20 \%$ to $30 \%$. It is known that it is not appropriate to use mean statistics in skewed data, particularly when there is a strong association between dispersed cases and final outcomes. Therefore, dispersity may be the reason why the neuroprotective effect of hypothermia is not significant in the meta-analysis. It is concluded that insignificant difference between $\mathrm{CPB}$ time is not sufficient to compare occurrence of POCD 
between groups, which is why studies need to be stratified for the CPB time as well.

Meta-regression showed that mild temperature difference $\left(-3^{\circ} \mathrm{C}\right)$ is related with a lower POCD in hypothermic group, meaning that mild hypothermic protocol $\left(34^{\circ} \mathrm{C}\right)$ showed a lower POCD in comparison with deep hypothermic protocol $\left(>34^{\circ} \mathrm{C}\right)$. In fact, other hypothermic protocol did not offer any advantage over normothermic group. Figure $3 \mathrm{~A}$ shows that the mild temperature difference (about $3^{\circ} \mathrm{C}$ ) is significantly associated with a lower rate of POCD, meaning that the neuroprotective effect of hypothermia decreases as temperature difference between normothermic and hypothermic group rises. This is in line with other studies showing that modest reductions in temperature have been shown to protect brain neurons and decrease lactate production. ${ }^{35}$ Ooboshi et al. indicated that mild brain hypothermia $\left(3^{\circ} \mathrm{C}\right.$ reduction) may be superior to attenuate effluxes of excitatory amino acids and protect of hippocampal neurons. ${ }^{28}$ Besides, a meta-analysis of the relationships between hypothermia and the neuroprotection effect after cardiac arrest showed that mild hypothermia $\left(32-34^{\circ} \mathrm{C}\right)$ improves neurological outcomes in resuscitated patients from cardiac arrest. ${ }^{29}$

Different time periods are used to examine cognitive status among RCTs (7 days, 4-6 weeks, 3 months, or longer). The follow-up period after $\mathrm{CPB}$ surgery ranged from 4 days to 5 years in the current studies. In the first week, the occurrence of POCD is at the highest rate, followed by a significant reduction over the first 6 months. ${ }^{3,6,12,36}$ In this line, Cormack et al. indicated that a trend developed towards a decrease in the occurrence of POCD across all 4 neuropsychological tests in the $1^{\text {st }}$ year following $\mathrm{CPB}$ surgery. ${ }^{1}$ They also mentioned there is a decline in half of the neuropsychological tests in the $1^{\text {st }}$ week after CPB surgery, specifically in those tests that assess psychomotor function. ${ }^{1}$ Apart from that, the practice effect may occur during the neuropsychological tests, so the effect size may be confounded by the patients' scores which showed practice effects over multiple testing sessions. ${ }^{2}$ However, the practice effect may improve scores towards a decline in the occurrence of POCD. Brown et al. also reported that there is a consistent reduction in the microemboli load as time passes after CPB surgery, ${ }^{6}$ implying that there may be multiple factors associated with trend towards decline in POCD.

The variability in the definition of POCD may contribute to the heterogeneity among RCTs. ${ }^{37}$ A 1 SD decline in the postoperative score in comparison to the preoperative score is the most commonly used measure to define POCD in the literature. It uses a fixed amount of decline for each patient and is specific to each study. It also estimates between the higher (20\% drop) and lower (IRs) estimates of cognitive decline. Therefore, a 1 SD decline criterion may miss up to $1 / 3$ of the occurrences of POCD. ${ }^{37}$ Finally, this error may be ignored because both hypothermic and normothermic groups have been classified with same methodology in each individual study, so it may have no significant impact on the effect size. Neuropsychological testing is still considered as the main method for the measurement of POCD. Therefore, the result must be interpreted cautiously because the neurocognitive assessment is accompanied by inherent variations in measuring POCD.

Patients lost to follow-up ranged from $1.3 \%$ to $73.73 \%$ in the current review. Loss to follow-up was not associated with the occurrence of POCD through meta-regression; in Heyer et al., it was $73.73 \%$ in early assessment. ${ }^{23}$ The validity of studies is questionable if more than $20 \%$ of patients are lost to follow-up. ${ }^{38}$ After exclusion of the study by Heyer et al., there was no significant association between hypothermia and POCD and the association between $\mathrm{CPB}$ time and occurrence of POCD also remained significant. ${ }^{23}$ These authors reported that loss to follow-up rate does not significantly differ between groups in order to lessen the bias caused by a high drop-out rate.

Further investigation of the effect of hypothermia on POCD with a focus on important moderators is necessary to provide a better understanding of the profile risks. We could not analyze the association between the patient's profile risk factors such as age, gender, DM, HTN and rLVEF, and POCD, since the studies did not report the patient's profile risk factors completely.

Several factors may be associated with cognitive deficit, but very few have been proven to have the significant effect on cognitive deficit as much as CPB surgery time. A higher occurrence of POCD is reported among patients undergoing CABG surgery with valve plasty. ${ }^{6}$ All included RCTs did not recruit patients with valve plasty surgery, which is why it is not considered as a source of bias in our metaanalysis. All included studies used on-pump CABG with conventional extracorporeal bypass, so the results may not be confounded by surgery technique. Generally, on- and off-pump CABG groups do not differ in terms of the occurrence of cognitive deficit. A meta-analysis reported comparing off-pump compared to on-pump CABG does not show any significant effect on POCD. ${ }^{14,39}$

There are several limitations of this meta-analysis. Studies have been conducted from 1994 to 2007; therefore, it was impossible to contact all the authors for additional data. The skewness of CPB time could be calculated if we had access to raw data and median CPB time. Further investigation of the effect of hypothermia on POCD with focus on CPB time is necessary. Stepwise regression model showed that $\mathrm{CPB}$ time and temperature contribute to $43.8 \%$ of the overall results, so patient risk profiles may be involved in the neuroprotective effect of hypothermia on POCD..$^{5,12}$

\section{Conclusions}

Meta-analysis of the available evidence showed that POCD is a common event among CABG patients. However, hypothermia was not significantly associated with a lower 
occurrence of POCD; mild hypothermia may be more effective than deep hypothermia in decreasing the risk of POCD. Shorter CPB time was associated with a lower occurrence of POCD, so the neuroprotective effect of hypothermia on POCD may be attenuated by prolonged $\mathrm{CPB}$ time.

\section{ORCID iDs}

Valiollah Habibi (1) https://orcid.org/0000-0002-5892-8683

Mohammad Reza Habibi (1) https://orcid.org/0000-0001-7495-2854

Ali Habibi (1) https://orcid.org/0000-0002-9145-482X

Amir Emami Zeydi @ ib https://orcid.org/0000-0001-8984-3298

\section{References}

1. Cormack F, Shipolini A, Awad W, et al. A meta-analysis of cognitive outcome following coronary artery bypass graft surgery. Neurosci Biobehav Rev. 2012;36(9):2118-2129.

2. Gholipour Baradari A, Habibi M, Habibi V, Nouraei S. Administration of lidocaine to prevent cognitive deficit in patients undergoing coronary artery bypass grafting and valve plasty: A systematic review and meta-analysis. Expert Rev Clin Pharmacol. 2017:10(2):179-185.

3. van Dijk D, Keizer A, Diephuis J, Durand C, Vos L, Hijman R. Neurocognitive dysfunction after coronary artery bypass surgery: A systematic review. J Thorac Cardiovasc Surg. 2000;120(4):632-639.

4. Ho K, Tan J. Benefits and risks of maintaining normothermia during cardiopulmonary bypass in adult cardiac surgery: A systematic review. Cardiovasc Ther. 2011;29(4):260-279.

5. Haddock C, Poston W, Taylor J. Neurocognitive sequelae following coronary artery bypass graft: A research agenda for behavioral scientists. Behav Modif. 2003;27(1):68-82.

6. Brown W, Moody D, Challa V, Stump D, Hammon J. Longer duration of cardiopulmonary bypass is associated with greater numbers of cerebral microemboli. Stroke. 2000;31(3):707-713.

7. Gutsche J, Feinman J, Silvay G, et al. Practice variations in the conduct of hypothermic circulatory arrest for adult aortic arch repair: Focus on an emerging European paradigm. Heart Lung Vessel. 2014;6(1):43-51.

8. Tan AMY, Amoako D. Postoperative cognitive dysfunction after cardiac surgery. Continuing Education in Anaesthesia, Critical Care \& Pain. 2013;13(6):218-223.

9. Rees K, Beranek-Stanley M, Burke M, Ebrahim S. Hypothermia to reduce neurological damage following coronary artery bypass surgery. Cochrane Database Syst Rev. 2001;1:CD002138.

10. Grigore A, Murray C, Ramakrishna H, Djaiani G. A core review of temperature regimens and neuroprotection during cardiopulmonary bypass: Does rewarming rate matter? Anesth Analg. 2009;109(6):1741-1751.

11. van Harten A, Scheeren T, Absalom A. A review of postoperative cognitive dysfunction and neuroinflammation associated with cardiac surgery and anaesthesia. Anaesthesia. 2012;67(3):280-293.

12. Patel N, Minhas J, Chung E. Risk factors associated with cognitive decline after cardiac surgery: A systematic review. Cardiovasc Psychiatry Neurol. 2015;2015:370612.

13. Shaaban Ali M, Harmer M, Kirkham F. Cardiopulmonary bypass temperature and brain function. Anaesthesia. 2005;60(4):365-372.

14. Hassani S, Alipour A, Darvishi Khezri H, et al. Can Valeriana officinalis root extract prevent early postoperative cognitive dysfunction after CABG surgery? A randomized, double-blind, placebo-controlled trial. Psychopharmacology (Berl). 2015;232(5):843-850.

15. Engelman R, Baker R, Likosky D, et al. The Society of Thoracic Surgeons, The Society of Cardiovascular Anesthesiologists and The American Society of ExtraCorporeal Technology. Clinical practice guidelines for cardiopulmonary bypass: Temperature management during cardiopulmonary bypass. Ann Thorac Surg. 2015;100(2):748-757.

16. Jadad A, Moore R, Carroll D, et al. Assessing the quality of reports of randomized clinical trial: Is blinding necessary? Control Clin Trials. 1996;17(1):1-12.

17. Borenstein $\mathrm{M}$, Hedges L, Higgins J, Rothstein $\mathrm{H}$. Introduction to MetaAnalysis. Hoboken, NJ: John Wiley \& Sons; 2009:187-203.

18. Tarsilla M. Cochrane handbook for systematic reviews of interventions. J Multidiscip Eval. 2010;6(14):142-148.
19. Mirhaghi A, Mazlom R, Heydari A, Ebrahimi M. The reliability of the Manchester Triage System (MTS): A meta-analysis. J Evid Based Med. 2017;10(2):129-135.

20. McLean RF, Wong BI, Naylor CD, et al. Cardiopulmonary bypass, temperature, and central nervous system dysfunction. Circulation. 1994; 90(5 Pt 2):II250-II255.

21. Mora CT, Henson MB, Weintraub WS, et al. The effect of temperature management during cardiopulmonary bypass on neurologic and neuropsychologic outcomes in patients undergoing coronary revascularization. J Thorac Cardiovasc Surg. 1996;112(2):514-522.

22. Regragui I, Birdi I, Izzat M, et al. The effects of cardiopulmonary bypass temperature on neuropsychologic outcome after coronary artery operations: A prospective randomized trial. J Thorac Cardiovasc Surg. 1996;112(4):1036-1045.

23. Heyer EJ, Adams DC, Delphin E, et al. Cerebral dysfunction after coronary artery bypass grafting done with mild or moderate hypothermia. J Thorac Cardiovasc Surg. 1997;114(2):270-277.

24. Plourde G, Leduc AS, Morin JE, et al. Temperature during cardiopulmonary bypass for coronary artery operations does not influence postoperative cognitive function: A prospective, randomized trial. J Thorac Cardiovasc Surg. 1997;114(1):123-128.

25. Kaukinen L, Porkkala H, Kaukinen S, et al. Release of brain-specific creatine kinase and neuron-specific enolase into cerebrospinal fluid after hypothermic and normothermic cardiopulmonary bypass in coronary artery surgery. Acta Anaesthesiol Scand. 2000;44(4): 361-368.

26. Boodhwani M, Rubens F, Wozny D, Rodriguez R, Nathan HJ. Effects of sustained mild hypothermia on neurocognitive function after coronary artery bypass surgery: A randomized, double-blind study. J Thorac Cardiovasc Surg. 2007;134(6):1443-1452.e1.

27. Nathan HJ, Rodriguez R, Wozny D, et al. Neuroprotective effect of mild hypothermia in patients undergoing coronary artery surgery with cardiopulmonary bypass: Five-year follow-up of a randomized trial. J Thorac Cardiovasc Surg. 2007;133(5):1206-1211.

28. Ooboshi $\mathrm{H}$, Ibayashi S, Takano K, et al. Hypothermia inhibits ischemiainduced efflux of amino acids and neuronal damage in the hippocampus of aged rats. Brain Res. 2000;884(1):23-30.

29. Holzer M, Bernard SA, Hachimi-Idrissi S, Roine RO, Sterz F, Müllner M; Collaborative Group on Induced Hypothermia for Neuroprotection After Cardiac Arrest. Hypothermia for neuroprotection after cardiac arrest: Systematic review and individual patient data meta-analysis. Crit Care Med. 2005;33(2):414-418.

30. Utley J, Leyland S, Johnson $\mathrm{H}$, et al. Correlation of preoperative factors, severity of disease, type of oxygenator and perfusion times with mortality and morbidity of coronary bypass. Perfusion. 1991;6:15-22.

31. Åberg T, Kihlgren M. Cerebral protection during open-heart surgery. Thorax. 1973;32(5):525-533.

32. Kuroda Y, Uchimoto R, Kaieda R, et al. Central nervous system complications after cardiac surgery: A comparison between coronary artery bypass grafting and valve surgery. Anesth Analg. 1993;76(2):222-227.

33. Salis $\mathrm{S}$, Mazzanti V, Merli G, et al. Cardiopulmonary bypass duration is an independent predictor of morbidity and mortality after cardiac surgery. J Cardiothorac Vasc Anesth. 2008;22(6):814-822.

34. Bucerius J, Gummert JF, Borger MA, et al. Stroke after cardiac surgery: A risk factor analysis of 16,184 consecutive adult patients. Ann Thorac Surg. 2003;75(2):472-478.

35. Berntman L, Welsh FA, Harp JR. Cerebral protective effect of lowgrade hypothermia. Anesthesiology. 1981;55(5):495-498.

36. Newman MF, Kirchner JL, Phillips-Bute B, et al; Neurological Outcome Research Group and the Cardiothoracic Anesthesiology Research Endeavors Investigators. Longitudinal assessment of neurocognitive function after coronary-artery bypass surgery. $N$ Engl I Med. 2001;344(6):395-402.

37. Mahanna EP, Blumenthal JA, White WD, et al. Defining neuropsychological dysfunction after coronary artery bypass grafting. Ann Thorac Surg. 1996;61(5):1342-1347.

38. Dettori J. Loss to follow-up. Evid Based Spine Care J. 2011:2(1):7-10.

39. Marasco S, Sharwood L, Abramson M. No improvement in neurocognitive outcomes after off-pump versus on-pump coronary revascularisation: A meta-analysis. Eur J Cardiothorac Surg. 2008;33(6):961-970. 